\title{
HMG-CoA Reductase Inhibitory Activity of Leaf-associated Fungi
}

\author{
Roland M. Hipol, ${ }^{1}$ Regina B. Hipol, ${ }^{2}$ Mavis Colleen P. Fabian, ${ }^{3}$ Joseph S. Sasotona ${ }^{3}$ and Christine C. Hernandez ${ }^{3}$ \\ ${ }^{1}$ Department of Biology, College of Science, University of the Philippines Baguio \\ ${ }^{2}$ Department of Pharmacy, School of Natural Sciences, Saint Louis University \\ ${ }^{3}$ Institute of Chemistry, College of Science, University of the Philippines Diliman
}

\begin{abstract}
Background. Cholesterol is an important biomolecule for cells. However, because of certain lifestyle, the amount of cholesterol can increase beyond what our bodies can consume. Increased intake may result in the accumulation of cholesterol in the blood leading to atherosclerosis that can lead to congestive heart failure. With the use of statins, cholesterol levels are reduced therefore lowering the risk for this disease. It does so by inhibiting the hydroxymethylglutaryl-coenzyme A (HMG-CoA) reductase, the first committed enzyme in cholesterol biosynthesis. In light of the increasing cases of hypercholesterolemia, there is a need to discover novel classes of HMG-CoA inhibitors.
\end{abstract}

Objective. The primary objective of this study was to screen extracts of leaf-associated fungi for their capacity to inhibit hydroxymethylglutaryl-coenzyme A (HMG-CoA) reductase, the first committed enzyme in cholesterol biosynthesis.

Methods. Eight (8) plants were sampled for the fungal epiphytes and endophytes on their leaves. The fungal isolates were identified by sequencing the internal transcribed region (ITS) of the $18 \mathrm{~S}$ rDNA. All fungal isolates were cultured in 20 flasks containing $300 \mathrm{~mL}$ potato dextrose broth at $30^{\circ} \mathrm{C}$ for 14 days. The culture broths were then subjected to reduction in volume using a rotary evaporator. HMG-CoA reductase inhibition assay was carried out using the ethyl acetate extracts from the culture broths. Reagent grade pravastatin was used as the positive control.

Results. There were a total of sixty-six (66) fungal taxa that were tested for their capacity to inhibit the enzyme HMG-CoA reductase. Of the 50 that tested positive, there were 25 families represented and 5 isolates were of uncertain status taxonomically (incertae sedis). There were also nine classes that were represented and the class with the most number of isolates was Sordariomycetes. The highest percentage inhibition was observed in Pestalotiopsis lespedezae with $65.01 \%$ inhibition. The other fungi with notable inhibitory activities were Colletotrichum cymbidiicola (64.16\%) and Schizophyllum commune (57.85\%).

Conclusions. This study may be the first report of production of HMG-CoA reductase inhibitors in the genus Colletotrichum and in the other leaf-associated fungal taxa that tested positive for HMG-CoA reductase inhibition. It is therefore very promising that a novel statin compound, or even a new class of substances with antihypercholesterolemic bioactivity may be discovered from these leaf-associated fungal isolates. The crude extracts of these fungi are prime candidates for downstream fractionation for the subsequent isolation and structure elucidation of the bioactive compound.

Key Words: HMG-CoA reductase inhibitor, cholesterol, statin, fungal endophyte, fungal epiphyte

\section{INTRODUCTION}

Corresponding author: Roland M. Hipol, PhD

Department of Biology

College of Science

University of the Philippines Baguio

Gov. Pack Rd., Baguio City 2600, Philippines

Email: rmhipol@up.edu.ph
Cholesterol is a very important biomolecule for the survival of cells. Around $70 \%$ of the cholesterol in our bodies is produced through the biosynthetic pathway observed in our cells and the rest should come from the diet. ${ }^{1}$ Because of certain lifestyle, the amount of cholesterol, especially 
bad cholesterol in the form of low and very low-density lipoproteins, is beyond what our bodies can consume. This condition is referred to as hypercholesterolemia, which is the accumulation of cholesterol in the blood leading to atherosclerosis. ${ }^{2}$ Ultimately, this may lead to congestive heart failure. ${ }^{3}$ Hypercholesterolemia is a specific condition of hyperlipidemia. Hyperlipidemia also includes elevated plasmatic concentrations of triglycerides ${ }^{4}$, which is beyond the scope of this research.

For people with very high levels of cholesterol, decrease in dietary intake alone is not enough to achieve the desired level. ${ }^{1}$ Current management of this condition uses the drug class referred to as statins. Statins control the de novo synthesis of endogenous cholesterol. It does so by targeting the rate limiting step in the biosynthesis of cholesterol through the inhibition of the hydroxymethylglutaryl-coenzyme A (HMG-CoA) reductase, the first committed enzyme in cholesterol biosynthesis. ${ }^{3}$ The mode of inhibition is due to the structural similarity of $\mathrm{HMGCoA}$, the natural substrate of the enzyme, and the acid form of the statins. ${ }^{5}$ Because of its efficacy in the lowering of blood cholesterol, statins are one of the largest selling classes of drugs throughout the world. In the US alone, sales for statins in 2013 was at $\$ 16.9$ billion. $^{6}$

Statins may be divided into three different classes depending upon whether their synthesis is natural, semisynthetic, or totally synthetic. The two naturally occurring statins are lovastatin and mevastatin. Akira Endo, a Japanese researcher, first discovered mevastatin as he was investigating for new antimicrobial compounds. ${ }^{6,7} \mathrm{He}$ discovered this HMG-CoA reductase inhibitor from fermentation broths of Penicilliumcitrinum. The pharmaceutical company Merck discovered another statin named lovastatin from Aspergillus terreus. ${ }^{8}$ Currently, many fungi have been reported to produce statins. These include Monascus ruber, $M$. purpureus, $M$. pilosus, Aspergillus terreus, A. flavipes, A. fischeri, A. flavus, A. umbrosus, $A$. parasiticus, Acremonium chrysogenum, Penicillium funiculosum, Trichoderma viridae and T. longibrachiatum.

In addition to the principal natural statins, several related compounds such as monacolins and dihydromonacolins, which are isolated fungal intermediate metabolites, have also been characterized. ${ }^{3}$ It is in this regard that this research has been conducted. Aside from the above fungal taxa, other fungi may be promising sources of antihypercholesterolemic agents. In this study, various foliar epiphytes and endophytes were investigated if they have the capacity to inhibit the enzyme HMG-CoA reductase.

\section{METHODS}

\section{Plant Sampling}

Eight (8) plants were sampled for their fungal epiphytes and endophytes in this study. These were a bryophyte, Marchantia polymorpha L. (Marchantiaceae); 2 ferns, Dicranopteris linearis N. L. Burman (Gleicheniaceae) and
Cyathea contaminans Wall. ex Hook. (Cyatheaceae) (tree fern); a gymnosperm Pinus kesiya Royle ex Gordon (Pinaceae) (Benguet Pine); 2 angiosperm monocots, Phragmites australis(Cav.) Trin. Ex Steud (Poaceae) (common reed), and Cenchrus polystachios (L.) Morrone (Poaceae) (mission grass); and 2 angiosperm dicots, Tithonia diversifolia (Hemsly) A. Gray (Asteraceae) (Mexican sunflower) and Rubus fraxinifolius Poir. (Rosaceae) (pinit). These plants were selected to represent the taxonomic diversity of the plant kingdom; as well as in consideration to the availability of these plants for sampling.

\section{Fungal isolation, cultivation and extraction}

Epiphytic fungi were isolated following the method used by Perez et al. ${ }^{9}$ Leaf sections were obtained from healthy leaves from each of the eight plants that were collected. After collection, leaf sections were stored at $4^{\circ} \mathrm{C}$ for no more than 7-10 days prior to suspension of epiphyte propagules. These sections were placed in $10 \mathrm{~mL}$ of a $0.5 \%$ sterilized gelatin solution and vortexed for $30 \mathrm{~s}$ at high speed in order to dislodge conidia and fungal cells from the leaf surface. Afterward, $100 \mu \mathrm{L}$ of the vortexed solution was spread-plated onto potato dextrose agar (PDA) medium containing chloramphenicol (Chlorolem - $500 \mathrm{mg}$ capsule) in order to prevent bacterial growth. Plates that were seeded with the phyllosphere solution were allowed to incubate at $30^{\circ} \mathrm{C}$. After 14 days of incubation, the number of total number of fungal colonies was counted, and the morphology of each was recorded. Pure cultures of each unknown were prepared in PDA slants.

Endophytic fungi were isolated healthy looking individuals from each of the eight species mentioned previously. Their leaves were processed immediately. The leaves were washed with tap water. Approximately, 0.25 $\mathrm{cm}^{2}$ portions of the leaves were surfaced sterilized. The following was the sequence and duration for each step of surface sterilization: $95 \%$ ethanol for $1 \mathrm{~min}, 10 \% \mathrm{NaOCl}$ for 5 mins then $95 \%$ ethanol for 30 s. $^{10}$ These were rinsed in sterile water then blotted dry on sterile absorbent paper. The effectiveness of surface sterilization method was tested by imprinting the leaf tissues on PDA plates. After overnight incubation at $30^{\circ} \mathrm{C}$, there was no growth observed on these plates that proved that surface sterilization was effective. These segments were incubated under ambient conditions on PDA with chloramphenicol to inhibit bacterial growth, and Rose Bengal to slow down the growth of fungi especially the vigorous ones. ${ }^{11}$ The plates were incubated for 14 days at $30^{\circ} \mathrm{C}$. Each colony was re-isolated in PDA slants.

All fungal isolates were cultured in 20 flasks containing $300 \mathrm{~mL}$ potato dextrose broth at $30^{\circ} \mathrm{C}$ for 14 days. The culture broth was then subjected to reduction in volume using a rotary evaporator. The extracts were stored at $4^{\circ} \mathrm{C}$ until the HMG-CoA reductase inhibition assay was conducted. 


\section{Molecular identification of fungal isolates}

The mycelia of the fungal isolates from the broth culture were comminuted in a microcentrifuge tube. The fungal isolates were identified by sequencing the internal transcribed region (ITS) of the $18 \mathrm{~S}$ rDNA, using universal primers ITS1 (5'TCC GTA GAA CCT GCG G-3') for the forward primer and ITS-4 (TCC TCC GCT TAT TGA TAT GC') for the reverse primer. The PCR products were run on $1 \%$ agarose gel to check the amplification of the desired length which is about $550 \mathrm{bp}$. The PCR products were sent to $1^{\text {st }}$ Base Inc. of Singapore for cleaning and subsequent sequencing.

The Basic Local Alignment Sequence Tool using nucleotides (BLASTn) search program (http://www.ncbi. nlm.nih.gov/BLAST/) was used to look for nucleotide sequence homology for the 18s ITS (1/4) region for the fungal isolates for their identification.

\section{HMG-CoA reductase inhibition assay}

HMG-CoA reductase inhibition assay was carried out using a method based on the one used by Perchellet et al. ${ }^{12}$ with some modifications. The buffer that was used was at $\mathrm{pH} 7.4100 \mathrm{mM}$ phosphate buffer with $120 \mathrm{mM} \mathrm{KCl}, 1 \mathrm{mM}$ EDTA and 50mM DTT. Each sample was plated with $\mathrm{NADPH}$ at $67.5 \mu \mathrm{M}$ per well and HMG-CoA (substrate) at $25 \mu \mathrm{M}$ per well. The plate was shaken and incubated at $37^{\circ} \mathrm{C}$. The reaction was initiated using the enzyme HMG-CoA reductase at $0.012 \mu \mathrm{M}$ per well. The reaction was monitored by reading at $340 \mathrm{~nm}$ every 20 seconds for ten minutes. Reagent grade pravastatin at $20 \mu \mathrm{M}$ per well was used as the positive control. Assays with the treatments and the control were performed in triplicates.
The enzyme inhibition was calculated using the following equation:

$$
\% \text { Inhibition }=\left[1-\left(\frac{A_{\text {sample }}}{A_{\text {uninhibited }}}\right)\right] \times 100
$$

where $A_{\text {uninhibited }}$ is the activity of negative control (all assay components except sample or inhibitor), $\mathrm{A}_{\text {sample }}$ is the activity in the presence of sample/inhibitor. Samples having $\geq 50 \%$ inhibition were considered active.

\section{RESULTS AND DISCUSSION}

There were a total of sixty-six (66) fungal taxa that were tested for their capacity to inhibit the enzyme HMGCoA reductase. Table 1 summarizes the result of the assay done with the fungal isolates. Of the 50 that tested positive, there were 25 families represented and 5 isolates were of uncertain status taxonomically (incertaesedis). There were also nine classes that were represented and the class with the most number of isolates was Sordariomycetes.

Of the 66 fungal taxa tested, only 16 tested negative in the HMG-CoA reductase assay; 50 had positive values for the computed percentage inhibition of the enzyme. The highest percentage inhibition was observed in Pestalotiopsis lespedezae with $65.01 \%$ inhibition. The other fungi with notable inhibitory activity were: Colletotrichum cymbidiicola (64.16\%), Schizophyllum commune (57.85\%), Colletotrichum karstii (56.48\%), Fusarium solani (55.77\%), Acremonium alternatum (55.26\%), Colletotrichum novae-zelandiae (54.26\%), Alternaria burnsii (53.42\%), Rbizomucor variabilis (52.76\%) and Colletotrichum boninense (52.65\%). Figure 1 shows the percentage inhibition computed for these fungal taxa.

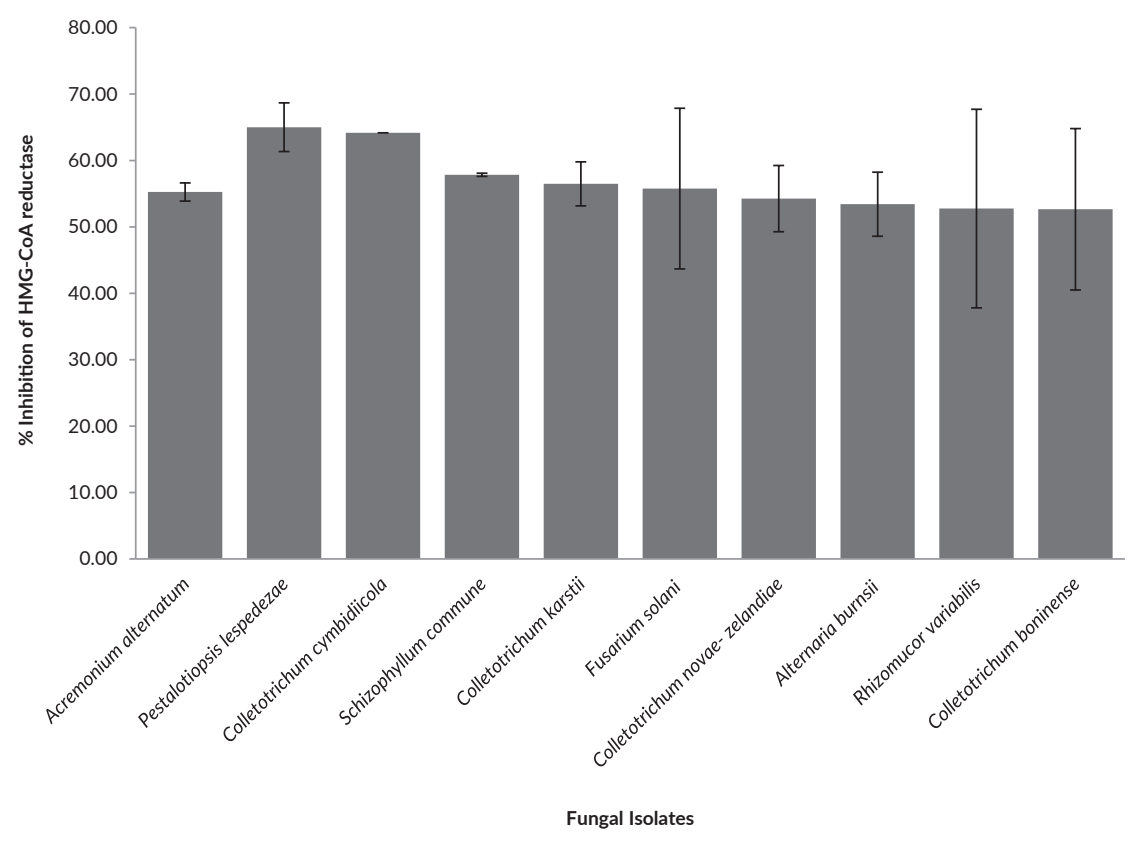

Figure 1. Computed percentage inhibition of the top performing fungal isolates. Error bars are the computed standard deviation of three replicates. 
Table 1. Fungal Isolates with HMG-CoA reductase inhibitory activity

\begin{tabular}{|c|c|c|c|}
\hline Fungal Isolate & \% Inhibition & Family & Class \\
\hline Acremonium alternatum & $55.26 \pm 1.37$ & incertaesedis & Sordariomycetes \\
\hline Alternaria burnsii & $53.42 \pm 4.82$ & Pleosporaceae & Dothideomycetes \\
\hline Ceriporia lacerata & $25.2 \pm 1.0$ & Phanerochaetaceae & Agaricomycetes \\
\hline Colletotrichum boninense & $52.65 \pm 12.15$ & Glomerellaceae & Sordariomycetes \\
\hline Colletotrichum cymbidiicola & $64.16 \pm 3.32$ & Glomerellaceae & Sordariomycetes \\
\hline Colletotrichumkarstii & $56.48 \pm 3.32$ & Glomerellaceae & Sordariomycetes \\
\hline Colletotrichum novae-zealandiae & $54.26 \pm 4.99$ & Glomerellaceae & Sordariomycetes \\
\hline Cryptococcus adaliensis & $33.06 \pm 9.17$ & Bulleribasidiaceae & Tremellomycetes \\
\hline Curvularias picifera & $14.66 \pm 3.94$ & Pleosporaceae & Dothideomycetes \\
\hline Curvularia trifolii & $20.69 \pm 34.29$ & Pleosporaceae & Dothideomycetes \\
\hline Hypocrea epimyces & $31.04 \pm 6.91$ & Hypocreaceae & Sordariomycetes \\
\hline Hypoxylon investiens & $37.85 \pm 3.05$ & Xylariaceae & Sordariomycetes \\
\hline Hypoxylon_pulicicidum & $35.04 \pm 0.01$ & Xylariaceae & Sordariomycetes \\
\hline Periconia byssoides & $19.89 \pm 2.43$ & Periconiaceae & Dothideomycetes \\
\hline Pestalotiopsis disseminata & $48.39 \pm 10.64$ & Sporocadaceae & Sordariomycetes \\
\hline Pestalotiopsis neglecta & $40.81 \pm 0.53$ & Sporocadaceae & Sordariomycetes \\
\hline Phialemonium dimorphosporum & $25.75 \pm 5.57$ & Cephalothecaceae & Sordariomycetes \\
\hline Scytalidium thermophilum & $14.07 \pm 1.41$ & incertaesedis & Leotiomycetes \\
\hline Xylaria intracolorata & $25.64 \pm 6.62$ & Xylariaceae & Sordariomycetes \\
\hline Cladosporium cladosporoides & $24.16 \pm 4.52$ & Cladosporiaceae & Dothideomycetes \\
\hline Corynespora casiicola & $28.74 \pm 2.19$ & Corynesporascaceae & Dothideomycetes \\
\hline Cryptococcus flavescens & $12.23 \pm 6.61$ & Bulleribasidiaceae & Tremellomycetes \\
\hline Diaportheeres & $28.33 \pm 18.5$ & Diaporthaceae & Sordariomycetes \\
\hline Dokmaia monthadangii & $50.92 \pm 15.52$ & incertaesedis & Dothideomycetes \\
\hline Fusarium solani & $55.77 \pm 12.1$ & Nectriaceae & Sordariomycetes \\
\hline Fusarium sporotrichioides & $12.55 \pm 9.26$ & Nectriaceae & Sordariomycetes \\
\hline Hypocrea lixii & $31.48 \pm 9.25$ & Hypocreaceae & Sordariomycetes \\
\hline Leptosphaerulina chartarum & $15.35 \pm 6.21$ & Didymellaceae & Dothideomycetes \\
\hline Leptosphaerulina trifolii & $24.55 \pm 2.72$ & Didymellaceae & Dothideomycetes \\
\hline Mucor hiemalis & $7.54 \pm 1.06$ & Mucoraceae & Zygomycetes \\
\hline Nectria_ipomoeae & $34.93 \pm 6.46$ & Nectriaceae & Sordariomycetes \\
\hline Pestalotiopsis lespedezae & $65.01 \pm 3.66$ & Sporocadaceae & Sordariomycetes \\
\hline Phoma herbarum & $42.95 \pm 6.48$ & Didymellaceae & Dothideomycetes \\
\hline Phomo psiscapsici & $12.61 \pm 1.77$ & Valsaceae & Sordariomycetes \\
\hline Pithomyces cynodontis & $10.47 \pm 3.87$ & Pleosporaceae & Dothideomycetes \\
\hline Pseudozyma_hubeiensis & $18.99 \pm 0.7$ & Ustilaginaceae & Ustilaginomycetes \\
\hline Pseudozyma_jejuensis & $23.41 \pm 9.89$ & Ustilaginaceae & Ustilaginomycetes \\
\hline Pyrenochaetain florescentiae & $17.03 \pm 3.61$ & Cucurbitariaceae & Dothideomycetes \\
\hline Rhizomucor variabilis & $52.76 \pm 1.96$ & Lichtheimiaceae & Zygomycetes \\
\hline Rhizosphaera macrospora & $33.92 \pm 2.54$ & Dothideaceae & Dothideomycetes \\
\hline Rhodotorula glutinis & $33.19 \pm 3.65$ & Sporidiobolaceae & Microbotryomycetes \\
\hline Rhodotorula graminis & $21.1 \pm 2.21$ & Sporidiobolaceae & Microbotryomycetes \\
\hline Rhodotorula_mucilaginosa & $19.72 \pm 5.98$ & Sporidiobolaceae & Microbotryomycetes \\
\hline Rosasphaeria moravica & $45.4 \pm 7.56$ & Niessliaceae & Sordariomycetes \\
\hline Schizophyllum commune & $57.85 \pm 0.24$ & Schizophyllaceae & Agaricomycetes \\
\hline Sydowia polyspora & $7.47 \pm 2.09$ & Dothioraceae & Dothideomycetes \\
\hline Trichosporon jirovecii & $6.79 \pm 2.28$ & Trichosporonaceae & Tremellomycetes \\
\hline Trichothecium roseum & $52.18 \pm 4.64$ & incertaesedis & Sordariomycetes \\
\hline Xylaria curta & $40.39 \pm 2.35$ & Xylariaceae & Sordariomycetes \\
\hline Yamadazymas colyti & $30.09 \pm 9.47$ & Debaryomycetaceae & Saccharomycetes \\
\hline
\end{tabular}


It is notable that all of the 50 fungal extracts tested positive against $\mathrm{HMG}-\mathrm{CoA}$ reductase despite them being crude aqueous or ethanolic extracts. However small, these crude extracts contained a compound/s that was able to inhibit the target enzyme. The $65.01 \%$ inhibition of HMG-CoA observed in Pestalotiopsis lespedezae is notable. Rather and Dhawan ${ }^{12,13}$ mentioned in their paper that the genus Pestalotiopsis produces the compound pestacin that has HMG-CoA inhibition capacity. This explains the inhibition activity of the extract against HMG-CoA. Another interesting isolate is Schizophyllum commune, with the third highest inhibition activity. Pushpa ${ }^{14}$ mentions that this basidiomycete produces lovastatin; thus the observed inhibitory activity. Fusarium solani exhibited the fifth best inhibitory activity. In the study of Pandey et al. ${ }^{15}$, they have found six Fusarium species, including F. solani, positive for lovastatin bioassay.

Of the families of fungi with multiple representatives, the most notable of them was the family Glomerellaceae represented by four species of Colletotrichum. What is interesting about this group is that all of the Colletotrichum species exhibited a consistent percentage inhibition of 52 to 64 percent. To-date, there has been no report of representatives of the genus Colletotrichum, nor of the family Glomerellaceae, that exhibit anti-hypercholesterolemic bioactivity. In the study of Bhargavi, Praveen and Savitha ${ }^{16}$, they found that the Colletotrichum Sp. they isolated as an endophyte did not possess the lovastatin biosynthetic genes $100 \mathrm{E}$ and $100 \mathrm{~F}$. This led them to conclude that the endophytic Colletotrichum, and other endophytes, do not have the capacity to produce the HMG-CoA reductase-inhibiting compound lovastatin. Base on the results of this study, HMG-CoA reductase inhibiting compounds may be discovered from fungal endophytes, in contrast to the findings of Bhargavi, Praveen and Savitha. ${ }^{16}$

This study may be the first report of production of HMG$\mathrm{CoA}$ reductase inhibitors in the genus Colletotrichum. In addition to this genus, there are thirteen other fungal genera in this study that were able to inhibit HMG-CoA reductase, albeit at different degrees. To the authors' knowledge, there is no literature that found anti-hypercholesterolemic activity in these taxa. It is therefore very promising to further study these plant-symbiotic fungi in the hope that a novel statin compound, or even a new class with antihypercholesterolemic bioactivity may be discovered from these. The crude extracts of these fungi are prime candidates for downstream fractionation for the subsequent isolation and structure elucidation of the bioactive compound.

\section{Statement of Authorship}

All authors participated in data collection and analysis, and approved the final version submitted.

\section{Funding Source}

This paper was funded by the Department of Science and Technology - Philippine Council for Health Research and Development (DOST-PCHRD).

\section{REFERENCES}

1. Lin SH, Chang DK, Chou MJ, Huang KJ, Shiuan D. Peptide inhibitors of human HMG-CoA reductase as potential hypocholesterolemia agents. Biochem Biophys Res Commun. 2015 Jan; 456(1):104-9. doi:10.1016/j.bbrc.2014.11.042.

2. Dikshit R, Tallapragada P. Bio-synthesis and screening of nutrients for lovastatin by Monascus sp. under solid-state fermentation. J Food Sci Technol. 2015 Oct; 52(10):6679-86. doi:10.1007/s13197-014-1678-y.

3. Manzoni M, Bergomi S, Rollini M, Cavazzoni V. Production of statins by filamentous fungi. Biotechnol Lett. 1999; 21: 253-7. doi: 10.1023/A:1005495714248.

4. Mbikay M. Therapeutic potential of Moringa oleifera leaves in chronic hyperglycemia and dyslipidemia: a review. Front Pharmacol. 2012 Mar; 3:24. doi:10.3389/fphar.2012.00024.

5. Manzoni M, Rollini M. Biosynthesis and biotechnological production of statins by filamentous fungi and application of these cholesterollowering drugs. Appl Microbiol Biotechnol. 2002 Apr; 58(5):555-64. doi:10.1007/s00253-002-0932-9.

6. Weintraub WS. Perspective on trends in statin use. JAMA Cardiol. 2017 Jan; 2(1):11-12. doi:10.1001/jamacardio.2016.4710.

7. Endo A. A historical perspective on the discovery of statins. Proc Jpn Acad Ser B Phys Biol Sci. 2010; 86(5):484-93.

8. Goswami S, Vidyarthi AS, Bhunia B, Mandal T. A review on lovastatin and its production. J Biochem Tech. 2012; 4(1):581-7.

9. Perez JL, French JV, Summy KR, Baines AD, Little CR. Fungal phyllosphere communities are altered by indirect interactions among trophic levels. Microb Ecol. 2009 May; 57(4):766-74. doi:10.1007/ s00248-008-9477-5.

10. Schulz B, Wanke U, Draeger S, Aust HJ. Endophytes from herbaceous plants and shrubs: effectiveness of surface sterilization methods. Mycol Res. 1993; 97(12):1447-50.

11. Ottow JC, Glathe H. Rose bengal-malt extract-agar, a simple medium for the simultaneous isolation and enumeration of fungi and actinomycetes from soil. Appl Microbiol. 1968 Jan; 16(1):170-1.

12. Perchellet JPH, Perchellet EM, Crow KR, Buszek KR, Brown $\mathrm{N}$, Ellappan S, et al. Novel synthetic inhibitors of 3-hydroxy-3methylglutaryl-coenzyme A (HMG-CoA) reductase activity that inhibit tumor cell proliferation and are structurally unrelated to existing statins. Int J Mol Med. 2009 Nov; 24(5):633-43.

13. Rather RA, Dhawan V. 2016. Natural Products of Fungal Endophytes and their Therapeutic Potential: A Focus on Cardiovascular Disease [Internet]. 2016 [cited 2018 Nov 8]. Available from: https://www. researchgate.net/profile/Dr_Riyaz_Ahmad/publication/311562209_ Natural_Products_of_Fungal_Endophytes_and_their_ Therapeutic_Potential_A_Focus_on_Cardiovascular_Disease/ links/584e63c008aecb6bd8cbef3c.pdf.

14. Pushpa H, Priyata H, Nomita Devi K, Onya N, Vijayalakshmi A, Ramesh DH. Screening of lovastatin (HMG-CoA reductase inhibitor) from edible wild mushrooms. Curr Res Environ Appl Mycol. 2016; 6(3):190-6. doi:10.5943/cream/6/3/6.

15. Pandey VV, Verma S, Gupta N, Varshney VK, Pandey A. Screening of different Fusarium species for bioassay of lovastatin. J Pharmacogn Phytochem. 2018; 7(5):127-39.

16. Bhargavi SD, Praveen VK, Savitha J. Screening of selected soil and endophytic fungi for lovostatin biosynthetic genes lovE and lovF. J Microb Biochem Technol. 2015; 7(6):334-7. doi:10.4172/19485948.1000235 .

\section{Author Disclosure}

All authors declared no conflicts of interest. 